\title{
Measurement of exclusive processes with CMS
}

\author{
Alexander BYLINKIN*† \\ Moscow Institute of Physics and Technology (MIPT), Moscow, Russia \\ E-mail: alexander.bylinkin@gmail.com
}

Exclusive vector meson photoproduction is studied in ultra-peripheral $\mathrm{pPb}$ collisions at $\sqrt{s_{N N}}=$ $5.02 \mathrm{TeV}$. The cross sections are measured as a function of the photon-proton centre-of-mass energy, extending the energy range explored by $\mathrm{H} 1$ and ZEUS Experiments at HERA. In addition, the differential cross sections $(d \sigma / d|t|)$, where $|t| \approx p_{T}^{2}$ is the squared transverse momentum of produced vector mesons, are measured and the slope parameters are obtained. The results are compared to previous measurements and to theoretical predictions.

We also report a measurement of the exclusive or quasi-exclusive $W^{+} W^{-}$production in pp collisions at $\sqrt{s}=8 \mathrm{TeV}(7 \mathrm{TeV})$ using data corresponding to an integrated luminosity of $19.7 \mathrm{fb}^{-1}$ $\left(5.5 \mathrm{fb}^{-1}\right)$, respectively. In this study, we look for any deviations that there might be from the Standard Model, and the results are used to set limits on the Anomalous Quartic Gauge Couplings.

Finally, the latest performance plots of combined CMS-TOTEM data are presented and prospects for further measurements are discussed.

XXV International Workshop on Deep-Inelastic Scattering and Related Subjects

3-7 April 2017

University of Birmingham, $U K$

* Speaker.

${ }^{\dagger}$ on behalf of the CMS Collaboration. 


\section{INTRODUCTION}

The exclusive production in proton-proton (or proton-ion) collisions, $p p(A) \rightarrow p+X+p(A)$, is characterized by the produced system $X$, where $X$ is emitted at central rapidities $y$ and the incident protons stay intact or dissociate without detection. Exclusive production in proton-proton collisions at central rapidities is usually described in terms of double pomeron exchange (DPE) processes, when the mass of the central system is not very large, or perturbatively, as in central exclusiveproduction approaches where the partonic structure of the pomeron (color singlet exchange with vacuum quantum numbers) is explicitly taken into account. In turn, proton-ion collisions allow to study high-energy photon-induced interactions with proton and/or ion beams, due to the high energy and large integrated luminosities available at the LHC. The CMS detector [1] provides a very wide range of opportunities to study both of these processes.

\section{EXCLUSIVE $\Upsilon$ PHOTOPRODUCTION IN P-PB COLLISIONS AT 5.02 TEV}

Exclusive photoproduction of heavy vector mesons (Figure 1, left) at very high photon-proton centre-of-mass energies $\left(W_{\gamma p}\right)$ can be studied in ultraperipheral collisions (UPC) of protons (ions). Recently, CMS [2], ALICE [3] and LHCb [4] presented their measurements of exclusive heavy vector meson photoproduction at the LHC. The process occurs through $\gamma p$ or $\gamma P b$ interactions via the exchange of two-gluons with no net color transfer and thus, at leading order (LO), the crosssection is proportional to the square of the gluon density in the target proton or ion. It provides a valuable probe of the gluon density at a small momentum fraction $x$, which is kinematically related to $W_{\gamma p}\left(x=\left(M_{\Upsilon} / W_{\gamma p}\right)^{2}\right)$.
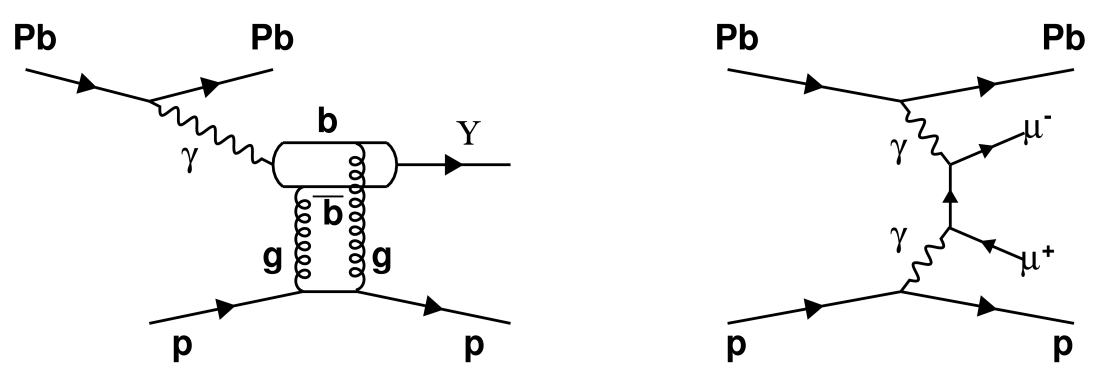

Figure 1: Diagrams representing exclusive $\Upsilon$ photoproduction (left), and exclusive dimuon QED continuum (right) in $\mathrm{pPb}$ collisions.

The exclusive photoproduction of $\Upsilon(1 S, 2 S, 3 S)$ has been measured in their dimuon decay channel in ultraperipheral collisions of protons and heavy ions $(\mathrm{pPb})$ with the CMS experiment at $\sqrt{s_{N N}}=5.02 \mathrm{TeV}$ for an integrated luminosity of $L_{\text {int }}=33 \mathrm{nb}^{-1}$ [5]. The photoproduction cross section for $\Upsilon(\mathrm{nS})$ was measured as a function of $W_{\gamma p}$ in the range $91<W_{\gamma p}<826 \mathrm{GeV}$, which corresponds to the rapidity of the $\Upsilon$ meson in the range $|y|<2.2$ and $x$ values are of the order $x \approx 10^{-4}$ to $x \approx 1.3 \cdot 10^{-2}$. The dependence of the elastic $\Upsilon$ photoproduction cross section on the squared $\Upsilon$ transverse momentum approximating the four-momentum transfer at the proton vertex $\left(|t| \approx p_{T}^{2}\right)$, can be parametrized with an exponential function $e^{-b|t|}$ at low values of $|t|$. The differential cross- 
section $d \sigma / d t$, has been measured in the range $|t|<1.0(\mathrm{GeV} / c)^{2}$ and the $b$-slope parameter was estimated.

The UPC events were selected by applying a dedicated trigger that selects at least one muon in each event and at least one to six tracks. To select the exclusive $\Upsilon(\mathrm{nS})$ events offline, two muon tracks originating from the same primary vertex in each event were used. The muons were selected with $p_{T}>3.3 \mathrm{GeV}$ and pseudorapidity $|\eta|<2.2$, in order to have high muon finding efficiency. The $p_{T}$ of the muon pair was selected between 0.1 to $1 \mathrm{GeV}$. The lower cut on the muon pair reduces the contamination from elastic QED background and the higher cut on the muon pair reduces the contamination from inelastic background (proton dissociation, inclusive $\Upsilon$, Drell-Yan). The rapidity of the muon pair is restricted to $|y|<2.2$.

These backgrounds were subtracted from data to get the exclusive signal. The background subtracted $|t|$ and $y$ distributions were used to measure the $b$ parameter and estimate the exclusive $\Upsilon$ photoproduction cross-section as a function of $W_{\gamma p}$, respectively. The distributions were first unfolded to the region $0.01<|t|<1(\mathrm{GeV} / c)^{2},|y|<2.2$, and muon $p_{T}^{\mu}>3.3 \mathrm{GeV}$, using the iterative Bayesian unfolding technique and its further extrapolation to zero transverse momenta by an acceptance correction factor.

The differential $d \sigma / d t$ cross section is extracted for the combined three $\Upsilon(n S)$ states as shown in Figure 2 (left), according to

$$
\frac{d \sigma_{\Upsilon}}{d|t|}=\frac{N^{\Upsilon(n S)}}{L \cdot \Delta|t|}
$$

where $|t|$ is approximated by the dimuon transverse momentum squared $p_{T}^{2}, N^{\Upsilon(n S)}$ denotes the background-subtracted, unfolded and acceptance-corrected number of signal events in each $|t|$ bin, $L$ is the integrated luminosity, and $\Delta|t|$ is the width of each $|t|$ bin. A value of $b=4.5 \pm 1.7$ (stat.) \pm 0.6 (syst.) $\mathrm{GeV}^{-2}$ is extracted from the exponential fit of the measured distribution. This result is in agreement with the value $b=4.3_{-1.3}^{+2.0}($ stat. $) \mathrm{GeV}^{-2}$ measured by the ZEUS experiment [6] for the photon-proton center-of-mass energy $60<W_{\gamma p}<220 \mathrm{GeV}$. The measured value of $b$ is also consistent with the predictions based on pQCD models [7].

The differential $\Upsilon(1 S)$ photoproduction cross section $d \sigma / d y$ is corrected by the feed-down contribution to the $\Upsilon(1 S)$ events and the branching ratio for muonic $\Upsilon(1 S)$ decays $B R=(2.48 \pm$ $0.05)$. The exclusive $\Upsilon(1 S)$ photoproduction cross section as a function of $W_{\gamma p}$ as shown in Figure 2 (right), is obtained by using,

$$
\sigma_{\gamma p \rightarrow \Upsilon(1 S) p}\left(W_{\gamma p}^{2}\right)=\frac{1}{\Phi} \frac{d \sigma_{\Upsilon(1 S)}}{d y}
$$

where $\Phi$ is the photon flux evaluated at the mean of the rapidity bin, estimated from STARLIGHT. The CMS data are plotted together with the previous measurements from H1 [8], ZEUS [9] and LHCb [4] data. It is also compared with different theoretical predictions of the JMRT model [7], factorized IPsat model [10], IIM [11, 12] and bCGC model [13]. As $\sigma\left(W_{\gamma p}\right)$ is proportional to the square of the gluon PDF of the proton and the gluon distribution at low Bjorken $x$ is well described by a power law, the cross section will also follow a power law. Any deviation from such trend would indicate a different behavior of the gluon density function. We fit a power-law $A \cdot(W / 400)^{\delta}$ with CMS data alone, which gives $\delta=0.96 \pm 0.43$ and $A=655 \pm 196$ and is shown by the black solid line. The extracted $\delta$ value is comparable to the value $\delta=1.2 \pm 0.8$, obtained by ZEUS [9]. 

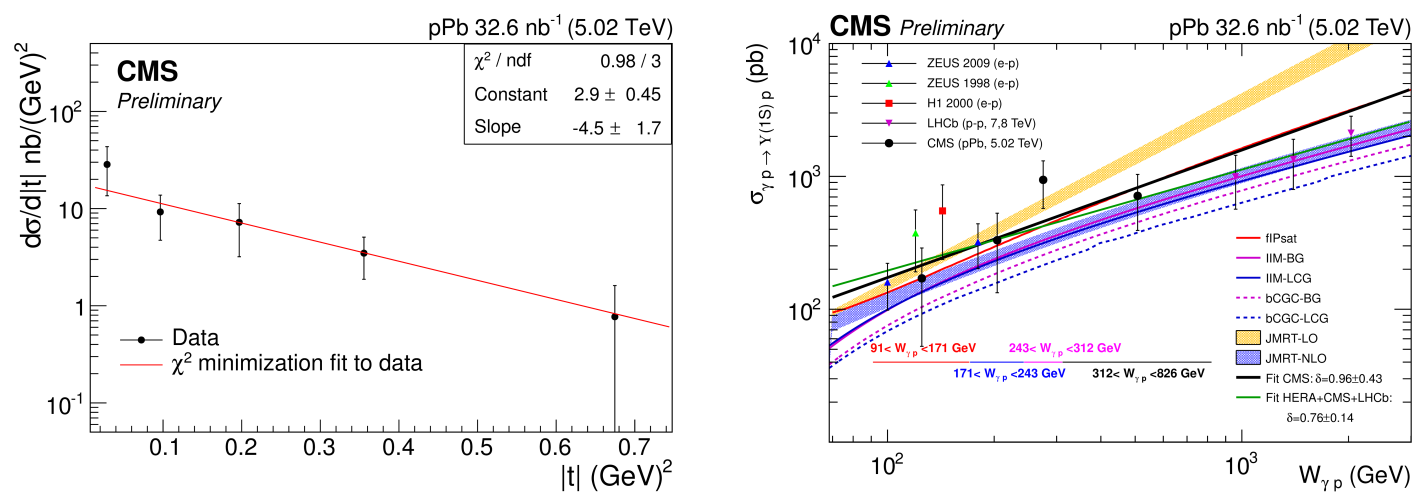

Figure 2: (Left) Differential $\Upsilon$ photoproduction cross section as a function of $|t|$ measured in pPb collisions at $\sqrt{s_{N N}}=5.02 \mathrm{TeV}$ in the dimuon rapidity region $|y|<2.2$. The solid line represents the result of a fit with an exponential function $N \cdot e^{b|t|}$. (Right) Cross section for exclusive $\Upsilon(1 S)$ photoproduction, $\gamma p \rightarrow \Upsilon(1 S) p$ as a function of photon-proton center-of-mass energy, $W_{\gamma p}[2]$.

\section{3. $W$ PAIR PRODUCTION}

The search for exclusive two-photon production of $W$ pairs is an opportunity to analyze processes that provide another study to test the standard model (SM). The measurement of the quartic $\gamma \gamma W W$ coupling can provide information if there is any deviation from the SM, which may be quantified by genuine anomalous quartic gauge couplings [14, 15] of dimension- 6 or dimension8. At the leading order, the contribution of the quartic, $t$-channel, and $u$-channel processes to the $\gamma \gamma \rightarrow W^{+} W^{-}$productions are shown in Figure 3 .
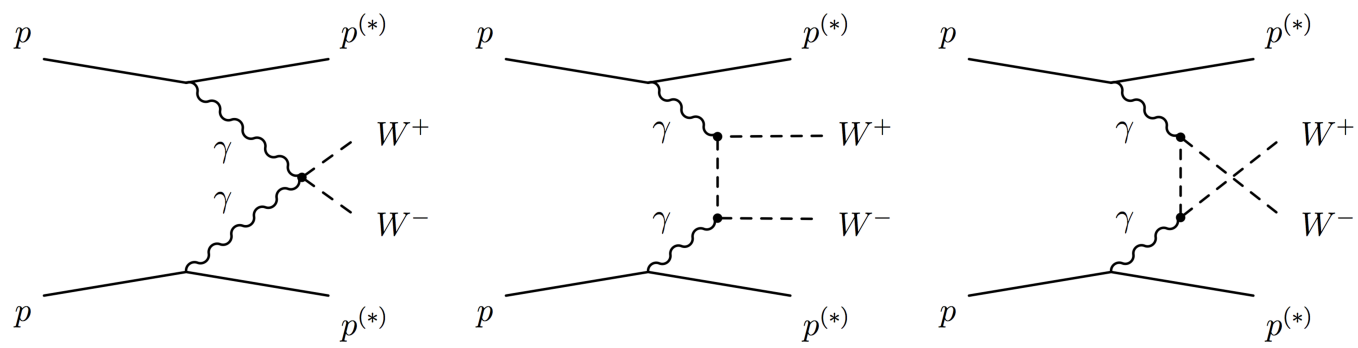

Figure 3: Quartic (left), $t$-channel (center), and $u$-channel (right) diagrams contributing to the $W^{+} W^{-}$ process at leading order in the SM [16].

The analysis has been carried out at $\sqrt{s}=7$ and $8 \mathrm{TeV}$ corresponding to an integrated luminosity of $5.5 \mathrm{fb}^{-1}$ and $19.7 \mathrm{fb}^{-1}$, respectively. The samples are analyzed to select exclusive events where the $W$ bosons decay into charged leptons. The signal production of $W$ pairs via $\mu^{ \pm} e^{\mp}$ channel, and $W^{+} W^{-} \rightarrow \mu^{+} \mu^{-}$or $W^{+} W^{-} \rightarrow e^{+} e^{-}$processes are dominated by Drell-Yan and $\gamma \gamma \rightarrow l^{+} l^{-}$ production. In order to reduce the contribution of backgrounds, the events are selected with a dedicated trigger requiring $p_{T}(l)>17(8) \mathrm{GeV}$ for the leading (subleading) lepton. The exclusivity requirement ensures that events have no additional tracks related to the dilepton vertex. Moreover, to suppress the $\tau^{+} \tau^{-}$backgrounds a transverse momentum of $p_{T}\left(l^{ \pm} l^{\mp}\right)>30 \mathrm{GeV}$ is applied.

After applying all the selection criteria, 2 events are observed at $7 \mathrm{TeV}$ compared to the expectation of $2.2 \pm 0.4$ signal and $0.84 \pm 0.14$ background, corresponding to an observed (expected) 
significance of $0.8 \sigma(1.86 \sigma)$. At $8 \mathrm{TeV}, 13$ events are observed compared to the expectation of $5.3 \pm 0.7$ signal and $3.9 \pm 0.6$ background events, corresponding to a mean expected signal significance of $2.1 \sigma$. By combining $7 \mathrm{TeV}$ and $8 \mathrm{TeV}$ results, the observed (expected) significance of $3.4 \sigma(2.8 \sigma)$ is obtained. The result as a cross section multiplied by the branching ratio to $\mu^{ \pm} e^{\mp}$ final states at $8 \mathrm{TeV}$ yields:

$$
\sigma_{p p} \rightarrow p^{(*)} W^{+} W^{-} p^{(*)} \rightarrow p^{(*)} \mu^{ \pm} e^{\mp} p^{(*)}=10.8_{-4.8}^{+5.1} \mathrm{fb} .
$$

Here $p^{(*)}$ denotes that the proton dissociative contribution is taken into account. Figure 4 (left) shows the $p_{T}$ distribution of $\mu \mathrm{e}$ with thirteen observed events. Furthermore, the transverse momentum $p_{T}\left(\mu^{ \pm} e^{\mp}\right)$ is used to search for the anomalous quartic gauge couplings, in the $\gamma \gamma \rightarrow W^{+} W^{-}$vertex, since any deviation from the SM prediction may indicate an evidence of new physics. Two bins (single bin) with transverse momentum with boundaries $p_{T}=30-130 \mathrm{GeV}$ and $p_{T}>130 \mathrm{GeV}\left(p_{T}>100 \mathrm{GeV}\right)$ are used to set the limit for $8 \mathrm{TeV}(7 \mathrm{TeV})$, respectively. Figure 4 (right) shows the two-dimensional limit of $a_{0}^{W}$ and $a_{C}^{W}$ anomalous parameters at $95 \%$ confidence level $[17,18,19]$. The exclusion region includes the $7 \mathrm{TeV}$ (outer contour), $8 \mathrm{TeV}$ (middle contour), and the combination of 7 and $8 \mathrm{TeV}$.
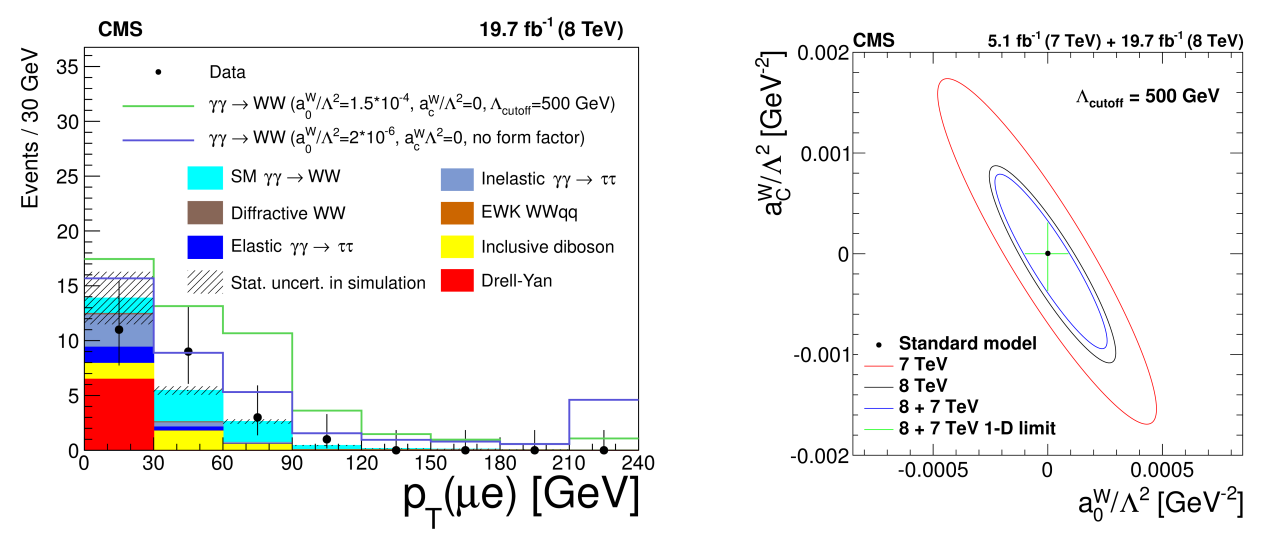

Figure 4: (Left) Transverse momentum distribution of the $\mu^{ \pm} e^{\mp}$ pair with the thirteen observed candidates. (Right) Two dimensional limit for the $a_{0}^{W}$ and $a_{C}^{W}$ anomalous parameters for $\Lambda_{\text {cutoff }}=500 \mathrm{GeV}$ in comparison to the limit measured at the LEP experiment [16].

\section{COMBINED CMS-TOTEM DATA}

In October 2015 about $0.4 \mathrm{pb}^{-1}$ of low-pileup $(\mu=0.06-0.15)$ data at $13 \mathrm{TeV}$ was collected in the joint CMS-TOTEM runs with $\beta^{*}=90 \mathrm{~m}$. Using this data, events with a central system in CMS and diffractive protons in TOTEM Roman Pots (RP) have been studied [20]. These events should have two scattered protons in the TOTEM Roman Pots, no activity in the TOTEM T2 telescopes and at least 5 clusters in the CMS pixel detector. CMS and TOTEM data were reconstructed separately and then merged offline event by event based on the same orbit and bunch crossing. In addition, a dedicated CMS track reconstruction tune was employed, to assure high track-finding efficiency for low- $p_{T}$ tracks and high vertex-finding efficiency for 2-track events. 
Transverse momenta, $p_{Y}$, of the scattered protons detected in the Roman Pots (TOTEM) versus transverse momenta of two pion tracks measured in the central tracking system (CMS) for the $p p \rightarrow p p \pi^{+} \pi^{-}$production is shown in Figure 5 (left). Events on the diagonal correspond to the exclusive $\pi^{+} \pi^{-}$production. Figure 6 (right) shows the projection of this distribution. One can notice a rather good resolution achieved, confirming that adding tagged protons from TOTEM greatly helps to select exclusive $p p \rightarrow p p \pi^{+} \pi^{-}$processes with no proton-dissociation.
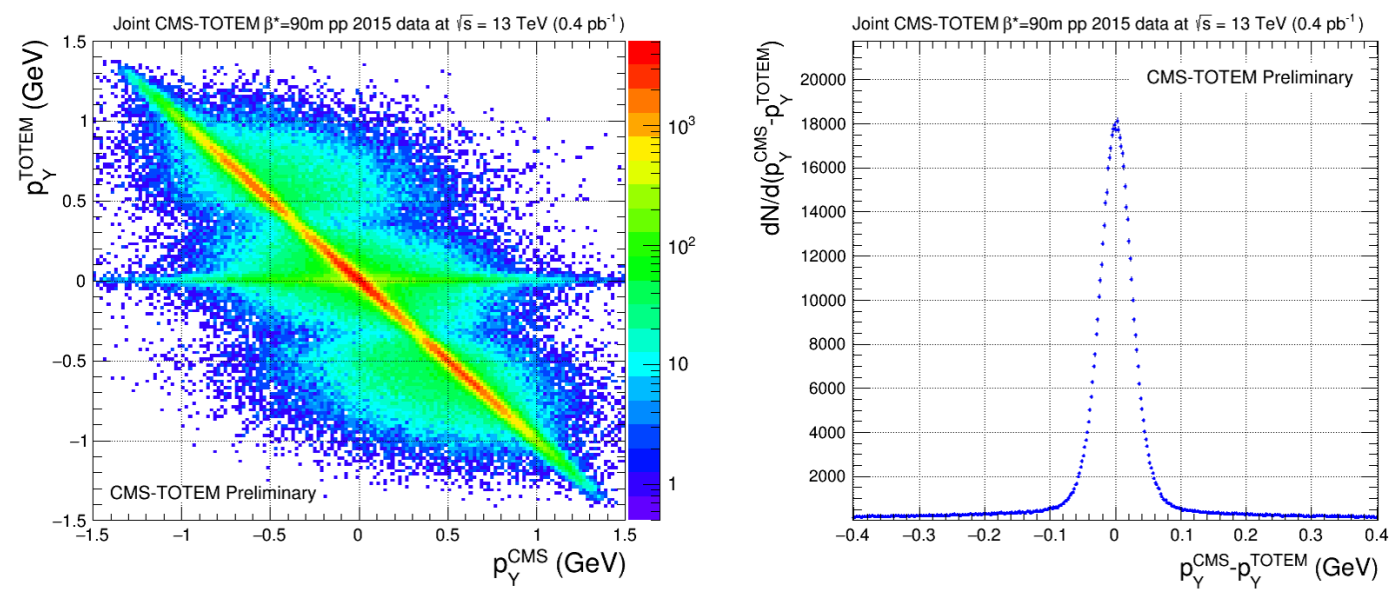

Figure 5: Transverse momenta, $p_{Y}$, of the scattered protons detected in Roman Pots (TOTEM) versus transverse momenta of two pion tracks measured in the central tracking system (CMS) for the $p p \rightarrow p p \pi^{+} \pi^{-}$ production (left) and its projection (right) [20].

\section{SUMMARY}

In these proceedings we reported several exclusive measurements from the CMS experiment. First, we presented the first measurement of the exclusive photoproduction of $\Upsilon(1 S, 2 S, 3 S)$ mesons in the $\mu^{+} \mu$ decay modes in ultraperipheral $\mathrm{pPb}$ collisions at $\sqrt{s_{N N}}=5.02 \mathrm{TeV}$, corresponding to an integrated luminosity of $33 \mathrm{nb}^{-1}$. The exclusive photoproduction cross sections have been measured as a function of the photon-proton center of mass energy, bridging a previously unexplored region between HERA and LHCb measurements. Our data are compatible with a powerlaw dependence of $\sigma\left(W_{\gamma p}\right)$, disfavouring faster rising predictions of LO pQCD. The spectral slope $b$ has been extracted, in agreement with earlier measurements.

Next, we studied the exclusive and quasi-exclusive $W^{+} W^{-}$production in pp collisions at a centre of mass energy of 7 and $8 \mathrm{TeV}$, corresponding to an integrated luminosity of $5.5 \mathrm{fb}^{-1}$ and $19.7 \mathrm{fb}^{-1}$, respectively. It is found that no significant deviation from the SM is observed.

Finally, the perspectives of using the combined CMS-TOTEM data have been discussed and it was shown to greatly help to select exclusive $p p \rightarrow p p \pi^{+} \pi^{-}$processes with no proton-dissociation background.

\section{References}

[1] S. Chatrchyan et al. [CMS Collaboration], JINST 3 (2008) S08004. doi:10.1088/1748-0221/3/08/S08004 
[2] CMS Collaboration, CMS-PAS-FSQ-13-009, http://cds.cern.ch/record/2147428

[3] B. B. Abelev et al. [ALICE Collaboration], Phys. Rev. Lett. 113 (2014) no.23, 232504

[4] R. Aaij et al. [LHCb Collaboration], JHEP 1509 (2015) 084

[5] CMS Collaboration, CMS-PAS-LUM-13-002, http://cds.cern.ch/record/1643269

[6] H. Abramowicz et al. [ZEUS Collaboration], Phys. Lett. B 708 (2012) 14

[7] S. P. Jones, A. D. Martin, M. G. Ryskin and T. Teubner, JHEP 1311 (2013) 085

[8] A. Aktas et al. [H1 Collaboration], Eur. Phys. J. C 46 (2006) 585

[9] S. Chekanov et al. [ZEUS Collaboration], Phys. Lett. B 680 (2009) 4

[10] T. Lappi and H. Mantysaari, Phys. Rev. C 83 (2011) 065202

[11] G. S. d. Santos and M. V. T. Machado, Phys. Rev. C 89 (2014) no.2, 025201

[12] G. Sampaio dos Santos and M. V. T. Machado, J. Phys. G 42 (2015) no.10, 105001

[13] V. P. Goncalves, B. D. Moreira and F. S. Navarra, Phys. Lett. B 742 (2015) 172

[14] S. Chatrchyan et al. [CMS Collaboration], JHEP 1201 (2012) 052

[15] S. Chatrchyan et al. [CMS Collaboration], JHEP 1307 (2013) 116

[16] V. Khachatryan et al. [CMS Collaboration], JHEP 1608 (2016) 119

[17] S. Chatrchyan et al. [CMS Collaboration], Phys. Lett. B 716 (2012) 30

[18] S. Chatrchyan et al. [CMS Collaboration], JHEP 1306 (2013) 081

[19] S. Chatrchyan et al. [CMS Collaboration], Phys. Rev. D 90 (2014) no.3, 032008

[20] CMS Collaboration, CMS-DP-2017-008, http://cds.cern.ch/record/2257969 